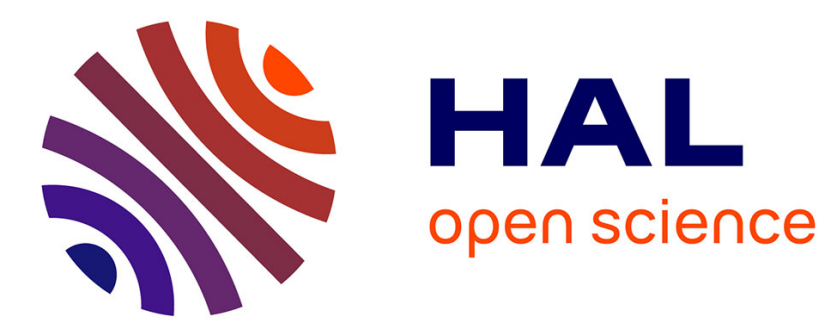

\title{
Comportement asymptotique à haute conductivité de l'épaisseur de peau en électromagnétisme
}

\author{
Monique Dauge, Erwan Faou, Victor Péron
}

\section{To cite this version:}

Monique Dauge, Erwan Faou, Victor Péron. Comportement asymptotique à haute conductivité de l'épaisseur de peau en électromagnétisme. Comptes Rendus. Mathématique, 2010, 348 (7-8), pp.385390. 10.1016/j.crma.2010.01.002 . hal-00423607

\section{HAL Id: hal-00423607 https://hal.science/hal-00423607}

Submitted on 12 Oct 2009

HAL is a multi-disciplinary open access archive for the deposit and dissemination of scientific research documents, whether they are published or not. The documents may come from teaching and research institutions in France or abroad, or from public or private research centers.
L'archive ouverte pluridisciplinaire HAL, est destinée au dépôt et à la diffusion de documents scientifiques de niveau recherche, publiés ou non, émanant des établissements d'enseignement et de recherche français ou étrangers, des laboratoires publics ou privés. 


\title{
Comportement asymptotique à haute conductivité de l'épaisseur de peau en électromagnétisme
}

\author{
Monique DAUGE ${ }^{\mathrm{a}}$, Erwan FAOU ${ }^{\mathrm{b}}$, Victor PÉRON $^{\mathrm{c}}$ \\ a IRMAR, Université de Rennes 1, Campus de Beaulieu, F-35042 Rennes Cedex, France \\ ${ }^{\mathrm{b}}$ INRIA, Projet Ipso, ENS Cachan Bretagne, Avenue Robert Schumann, F-35170 Bruz Cedex, France \\ ${ }^{\mathrm{c}}$ IRMAR, Université de Rennes 1, Campus de Beaulieu, F-35042 Rennes Cedex, France
}

\begin{abstract}
Résumé
On étudie un modèle tridimensionnel décrivant l'effet de peau en électromagnétisme. On commence par donner un développement asymptotique multi-échelle de la solution des équations de Maxwell en régime harmonique posées dans un domaine formé de deux matériaux, l'un diélectrique et l'autre fortement conducteur, avec une interface régulière entre les deux. Afin de mesurer l'effet de peau on introduit une fonction "épaisseur de peau" définie sur l'interface, ce qui généralise la quantité scalaire classique. On donne alors un développement asymptotique à haute conductivité pour cette fonction, ce qui met en évidence l'influence de la géométrie de l'interface sur l'épaisseur de peau.
\end{abstract}

\section{Abstract}

Asymptotic behavior at high conductivity of skin depth in electromagnetism

We study a three-dimensional model for the skin effect in electromagnetism. We first give a multiscale asymptotic expansion for the solution of the harmonic Maxwell equations set on a domain made of two materials, dielectric and highly conducting, with a regular interface between them. To measure the skin effect, we introduce a suitable skin depth function defined on the interface and generalizing the classical scalar quantity. We then prove an asymptotic expansion at high conductivity for this function, which exhibits the influence of the geometry of the interface on the skin depth.

Abridged English version Several works are devoted to asymptotic expansions at high conductivity of the electromagnetic field $(\mathbf{E}, \mathbf{H})$, solution of the Maxwell system

$$
\operatorname{curl} \mathbf{E}-i \omega \mu_{0} \mathbf{H}=0 \text { and } \operatorname{curl} \mathbf{H}+\left(i \omega \varepsilon_{0}-\underline{\sigma}\right) \mathbf{E}=\mathbf{j}
$$

Email addresses: monique.dauge@univ-rennes1.fr(Monique DAUGE), Erwan.Faoueinria.fr(Erwan FAOU), victor peron@univ-rennes1. fr (Victor PÉRON). 
with complementing boundary conditions when the interface $\Sigma$ is smooth: see [10,6,7] for plane interface and eddy current approximation, [4] for impedance boundary conditions and [9,1] for insulating and perfectly conducting boundary conditions. Here, $\mu_{0}$ is the magnetic permeability, $\varepsilon_{0}$ the electric permittivity, $\omega$ the angular frequency, $\mathbf{j}$ represents a current density, and $\underline{\sigma}$ the electrical conductivity. We assume that these equations are set in a domain $\Omega$ made up of two subdomains $\Omega_{+}$and $\Omega_{-}$in which the coefficient $\underline{\sigma}$ take two different values $\left(\sigma_{+}=0, \sigma_{-} \equiv \sigma\right)$. We suppose that the interface $\Sigma$ between $\Omega_{+}$and $\Omega_{-}$is an oriented smooth compact surface and that $\Sigma=\partial \Omega_{-}$.

Let $\left(y_{\alpha}, y_{3}\right)$ be a local normal coordinate system to the surface $\Sigma$ in a tubular neighborhood $\mathcal{U}_{-}$of $\Sigma$ in the conductor part $\Omega_{-}$. Here, $y_{\alpha}$ denote local coordinates on $\Sigma$ and $y_{3}=h$ is the distance to $\Sigma$, see [8,2]. It is possible to construct series expansions in powers of $\delta=\sqrt{\omega \varepsilon_{0} / \sigma}$ for the electromagnetic field solution of problem $(*)$ denoted by $\left(\mathbf{E}_{(\delta)}^{+}, \mathbf{H}_{(\delta)}^{+}\right)$in $\Omega_{+}$and $\left(\mathbf{E}_{(\delta)}^{-}, \mathbf{H}_{(\delta)}^{-}\right)$in $\Omega_{-}$:

$$
\begin{gathered}
\mathbf{E}_{(\delta)}^{+}(\mathbf{x}) \approx \sum_{j \geqslant 0} \delta^{j} \mathbf{E}_{j}^{+}(\mathbf{x}), \quad \mathbf{E}_{(\delta)}^{-}(\mathbf{x}) \approx \sum_{j \geqslant 0} \delta^{j} \mathbf{E}_{j}^{-}(\mathbf{x} ; \delta) \quad \text { and } \quad \mathbf{H}_{(\delta)}^{+}(\mathbf{x}) \approx \sum_{j \geqslant 0} \delta^{j} \mathbf{H}_{j}^{+}(\mathbf{x}), \mathbf{H}_{(\delta)}^{-}(\mathbf{x}) \approx \sum_{j \geqslant 0} \delta^{j} \mathbf{H}_{j}^{-}(\mathbf{x} ; \delta) \\
\text { with } \quad \mathbf{E}_{j}^{-}(\mathbf{x} ; \delta)=\chi\left(y_{3}\right) \mathbf{W}_{j}\left(y_{\beta}, \frac{y_{3}}{\delta}\right) \quad \text { and } \quad \mathbf{H}_{j}^{-}(\mathbf{x} ; \delta)=\chi\left(y_{3}\right) \mathbf{V}_{j}\left(y_{\beta}, \frac{y_{3}}{\delta}\right) .
\end{gathered}
$$

The function $\mathbf{y} \mapsto \chi\left(y_{3}\right)$ is a smooth cut-off with support in $\overline{\mathcal{U}}_{-}$and equal to 1 in a smaller tubular neighborhood of $\Sigma$. We derive explicitly the first profiles $\mathbf{W}_{0}, \mathbf{W}_{1}, \mathbf{W}_{2}, \mathbf{V}_{0}$, and $\mathbf{V}_{1}$. This result extends previous works about skin effect presented in $[6,7,10]$. Here, $\mathbf{W}_{j}=\left(\mathcal{W}_{\alpha, j}, w_{j}\right)$ is a 1 -form field in $\mathcal{U}_{-}$, and $\mathbf{V}_{j}=\left(\mathcal{V}_{j}^{\alpha}, v_{j}\right)$ is a vector field in $\mathcal{U}_{-}$. Tensorial calculus is performed by means of a normal parameterization of the intrinsic curl operator, see [9],

$$
(\nabla \times \mathbf{E})^{\alpha}=\epsilon^{3 \beta \alpha}\left(\partial_{3}^{h} E_{\beta}-\partial_{\beta} E_{3}\right) \quad \text { and } \quad(\nabla \times \mathbf{E})^{3}=\epsilon^{3 \alpha \beta} D_{\alpha}^{h} E_{\beta} \quad \text { on } \quad \Sigma_{h} .
$$

Here, $\epsilon$ is the Levi-Civita tensor, see [5,3], $D_{\alpha}^{h}$ is the covariant derivative on $\Sigma_{h}$, which is the surface contained in $\Omega_{-}$at a distance $h$ of $\Sigma$.

The surface components $\mathcal{W}_{\alpha}$ are distinct from those given in [4] which we denote here by $\widetilde{\mathcal{W}}_{\alpha}$. Actually these components are defined in distinct bases and are similar modulo the shifter, cf. [2, § 2.D]: We have $\mathcal{W}_{\alpha}(h) d y^{\alpha}(h)=$ $\widetilde{\mathcal{W}}_{\alpha}(h) d y^{\alpha}(0)$, with

$$
\widetilde{\mathcal{W}}_{\alpha}=\mathcal{W}_{\alpha}-h b_{\alpha}^{\beta} \mathcal{W}_{\beta} \quad \text { and } \quad \mathcal{W}_{\alpha}=\sum_{n \geqslant 0} h^{n}\left(b^{n}\right)_{\alpha}^{\beta} \widetilde{\mathcal{W}}_{\beta}
$$

where $d y^{\alpha}(h)$ and $d y^{\alpha}(0)$ are the local bases of the cotangent planes on $\Sigma_{h}$ and $\Sigma=\Sigma_{0} \subset \mathbb{R}^{3}$ respectively. Here, $b_{\alpha}^{\beta}=a^{\beta \sigma} b_{\sigma \alpha}$ (with the summation convention of repeated indices) and $\left(b^{n}\right)_{\alpha}^{\beta}=b_{\nu_{1}}^{\beta} b_{\nu_{2}}^{\nu_{1}} \cdots b_{\alpha}^{\nu_{n-1}}$, where $a^{\beta \sigma}$ and $b_{\sigma \alpha}$ are the inverse of the metric and curvature tensor on $\Sigma$ respectively.

In a one-dimensional model, when $\Omega_{-}$is a half-space, the classical skin depth parameter is given by $\ell(\sigma)=$ $\sqrt{2 / \omega \mu_{0} \sigma}$. In our situation, we extend this definition and introduce a characteristic and intrinsic length $\mathcal{L}\left(\sigma, y_{\alpha}\right)$ as the length where the field has decreased of a fixed rate, see Definition 4.1. This length $\mathcal{L}\left(\sigma, y_{\alpha}\right)$ depends on the conductivity $\sigma$ and each point in the interface $\Sigma$, and has the following behavior at high conductivity:

$$
\mathcal{L}\left(\sigma, y_{\alpha}\right)=\ell(\sigma)\left(1+\mathcal{H}\left(y_{\alpha}\right) \ell(\sigma)+\mathcal{O}\left(\sigma^{-1}\right)\right), \quad \sigma \rightarrow \infty,
$$

where $\mathcal{H}$ is the mean curvature of the surface $\Sigma$.

\section{Introduction}

On s'intéresse aux équations de Maxwell harmoniques données par les lois de Faraday et Maxwell-Ampère déterminant le champ électromagnétique $(\mathbf{E}, \mathbf{H})$

$$
\operatorname{rot} \mathbf{E}-i \omega \mu_{0} \mathbf{H}=0 \quad \text { et } \quad \operatorname{rot} \mathbf{H}+\left(i \omega \varepsilon_{0}-\underline{\sigma}\right) \mathbf{E}=\mathbf{j} .
$$


Ici $\underline{\sigma}$ représente la conductivité électrique, $\omega$ la fréquence angulaire, $\varepsilon_{0}$ la permittivité électrique du vide, $\mu_{0}$ la perméabilité magnétique du vide et $\mathbf{j}$ une source de courant. On suppose que ces équations sont posées dans un domaine $\Omega$ constitué de deux sous-domaines $\Omega_{+}$et $\Omega_{-}$dans lequel le coefficient $\underline{\sigma}$ prend deux valeurs différentes $\left(\sigma_{+}, \sigma_{-}\right)$. On suppose que $\partial \Omega \subset \partial \Omega_{+}$et on considère la condition de l'isolant parfait sur $\partial \Omega$

$$
\mathbf{E} \cdot \mathbf{n}=0 \quad \text { et } \quad \mathbf{H} \times \mathbf{n}=0,
$$

où $\mathbf{n}$ est la normale sortante sur $\partial \Omega$. La résolubilité des équations (1)-(2) ainsi que des estimations uniformes d'énergie ou de régularité lorsque $\sigma_{+}=0$ (matériau isolant ou diélectrique) et $\sigma_{-} \equiv \sigma$ tend vers l'infini (matériau fortement conducteur) sont étudiées dans [1] lorsque l'interface $\Sigma=\partial \Omega_{-}$est Lipschitz et pour une donnée $\mathbf{j} \in$ $\mathbf{H}_{0}(\operatorname{div}, \Omega)=\left\{\mathbf{u} \in \mathbf{L}^{2}(\Omega)=\mathrm{L}^{2}(\Omega)^{3} \mid \operatorname{div} \mathbf{u} \in \mathrm{L}^{2}(\Omega)\right.$ et $\mathbf{u} \cdot \mathbf{n}=0$ sur $\left.\partial \Omega\right\}$.

Dans la suite, on suppose que l'interface $\Sigma$ est une surface compacte orientable de classe $\mathcal{C}^{\infty}$ et on note $\mathbf{n}$ la normale sur $\Sigma$ rentrante dans $\Omega_{-}$.

En particulier, on peut définir localement des coordonnées normales (voir [8,2]) : soit $y_{\alpha}$ un système de coordonnées locales sur $\Sigma$ et $y_{3}$ la coordonnée normale représentant la distance à $\Sigma$. Alors $\mathbf{y}=\left(y_{\alpha}, y_{3}\right)$ sont des "coordonnées normales" à la surface $\Sigma$ dans un voisinage tubulaire $\mathcal{U}_{-}$de $\Sigma$ dans le conducteur $\Omega_{-}$.

On introduit le petit paramètre

$$
\delta=\sqrt{\omega \varepsilon_{0} / \sigma} .
$$

Ainsi, $\delta$ tend vers 0 lorsque $\sigma \rightarrow \infty$. Dans ce cadre d'étude, on peut calculer un développement en puissances de $\delta$ de la solution du problème (1), voir le paragraphe 2. On compare ce développement avec celui issu de [4] et obtenu par des calculs différentiels différents, voir le paragraphe 3 . Enfin, le but de cette note est de préciser le comportement de l'épaisseur de peau $\mathcal{L}\left(\sigma, y_{\alpha}\right)$ à la surface du conducteur $\Omega_{-}$lorsque $\delta \rightarrow 0$, voir le paragraphe 4 .

On note $a_{\alpha \beta}$ le tenseur métrique sur $\Sigma$ et $b_{\alpha \beta}$ le tenseur de courbure dans un système de coordonnées locales. La métrique permet de monter et descendre les indices. On note ainsi $b_{\alpha}^{\beta}=a^{\beta \sigma} b_{\sigma \alpha}$, où la répétition des indices covariants et contravariants correspond à la contraction des tenseurs, voir $[8,2]$.

\section{Développement asymptotique}

Soit $\mathbf{j} \in \mathbf{H}_{0}(\operatorname{div}, \Omega)$ tel que $\mathbf{j}=0$ dans $\Omega_{-}$. Sous l'hypothèse que $\omega$ n'est pas une fréquence propre du problème limite posé dans $\Omega_{+}$lorsque $\delta \rightarrow 0$ (voir [1,9]), il existe $\delta_{0}>0$ tel que pour tout $\delta \leqslant \delta_{0}$, le problème (1)-(2) admet une unique solution $\left(\mathbf{E}_{(\delta)}, \mathbf{H}_{(\delta)}\right) \in \mathbf{L}^{2}(\Omega)^{2}$. De plus, $\left(\mathbf{E}_{(\delta)}, \mathbf{H}_{(\delta)}\right)$ admet un développement asymptotique multi-échelle à tout ordre en puissances de $\delta,\left(\mathbf{E}_{(\delta)}^{+}, \mathbf{H}_{(\delta)}^{+}\right)$dans $\Omega_{+}$et $\left(\mathbf{E}_{(\delta)}^{-}, \mathbf{H}_{(\delta)}^{-}\right)$dans $\Omega_{-}$:

$$
\begin{gathered}
\mathbf{E}_{(\delta)}^{+}(\mathbf{x}) \approx \sum_{j \geqslant 0} \delta^{j} \mathbf{E}_{j}^{+}(\mathbf{x}) \quad \text { et } \quad \mathbf{H}_{(\delta)}^{+}(\mathbf{x}) \approx \sum_{j \geqslant 0} \delta^{j} \mathbf{H}_{j}^{+}(\mathbf{x}), \\
\mathbf{E}_{(\delta)}^{-}(\mathbf{x}) \approx \sum_{j \geqslant 0} \delta^{j} \mathbf{E}_{j}^{-}(\mathbf{x} ; \delta) \quad \text { avec } \quad \mathbf{E}_{j}^{-}(\mathbf{x} ; \delta)=\chi\left(y_{3}\right) \mathbf{W}_{j}\left(y_{\beta}, \frac{y_{3}}{\delta}\right), \\
\mathbf{H}_{(\delta)}^{-}(\mathbf{x}) \approx \sum_{j \geqslant 0} \delta^{j} \mathbf{H}_{j}^{-}(\mathbf{x} ; \delta) \quad \text { avec } \quad \mathbf{H}_{j}^{-}(\mathbf{x} ; \delta)=\chi\left(y_{3}\right) \mathbf{V}_{j}\left(y_{\beta}, \frac{y_{3}}{\delta}\right),
\end{gathered}
$$

où, d'après l'hypothèse spectrale sur $\omega,\left(\mathbf{E}_{0}^{+}, \mathbf{H}_{0}^{+}\right)$est l'unique solution du problème

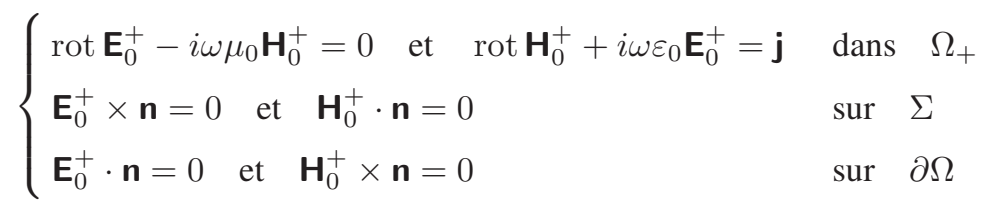


et dans la variable $Y_{3}=\delta^{-1} y_{3}$, les profils $\mathbf{W}_{j}$ et $\mathbf{V}_{j}$ sont définis sur $\Sigma \times \mathbb{R}_{+}$et satisfont

$$
\mathbf{W}_{j}\left(y_{\beta}, Y_{3}\right) \rightarrow 0 \quad \text { et } \quad \mathbf{v}_{j}\left(y_{\beta}, Y_{3}\right) \rightarrow 0 \quad \text { lorsque } \quad Y_{3} \rightarrow \infty \text {. }
$$

Dans (4), la fonction $\mathbf{y} \mapsto \chi\left(y_{3}\right)$ est une fonction de troncature régulière à support dans $\overline{\mathcal{U}}_{-}$et égale à 1 dans un voisinage de $\Sigma$ dans $\mathcal{U}_{-}$. Dans un système de coordonnées normales, on note $\mathcal{W}_{\alpha, j}$ les composantes surfaciques de $\mathbf{W}_{j}$, vu comme un champ de 1 -formes, et $w_{j}$ sa composante normale de sorte que $\mathbf{W}_{j}=\left(\mathcal{W}_{\alpha, j}, w_{j}\right)$. On note aussi $\mathcal{V}_{j}^{\alpha}$ les composantes surfaciques de $\mathbf{V}_{j}$, vu comme un champ de vecteurs, et $v_{j}$ sa composante normale. Dans la suite, on étend le système de coordonnées normales à $\mathcal{U}_{+}$, un voisinage tubulaire de $\Sigma$ dans $\Omega_{+}$. On note enfin $\mathrm{j}_{\alpha, k}\left(y_{\beta}\right):=\lambda^{-1}\left(\operatorname{rot} \mathbf{E}_{k}^{+} \times \mathbf{n}\right)_{\alpha}\left(y_{\beta}, 0\right)$ pour $k=0,1$ et $\lambda=\kappa e^{-i \pi / 4}\left(\kappa=\omega \sqrt{\varepsilon_{0} \mu_{0}}\right.$ est le nombre d'onde $)$. On a

$$
\begin{gathered}
\mathbf{W}_{0}\left(y_{\beta}, Y_{3}\right)=0 \\
\mathcal{W}_{\alpha, 1}\left(y_{\beta}, Y_{3}\right)=-\mathrm{j}_{\alpha, 0}\left(y_{\beta}\right) \mathrm{e}^{-\lambda Y_{3}} \quad \text { et } w_{1}=0, \\
\mathcal{W}_{\alpha, 2}\left(y_{\beta}, Y_{3}\right)=\left[-\mathrm{j}_{\alpha, 1}+\left(\lambda^{-1}+Y_{3}\right)\left(b_{\alpha}^{\sigma} \mathrm{j}_{\sigma, 0}-\mathcal{H} \mathrm{j}_{\alpha, 0}\right)\right]\left(y_{\beta}\right) \mathrm{e}^{-\lambda Y_{3}} \\
\text { et } w_{2}\left(y_{\beta}, Y_{3}\right)=-\lambda^{-1} D_{\alpha} \mathrm{j}_{0}^{\alpha}\left(y_{\beta}\right) \mathrm{e}^{-\lambda Y_{3}}
\end{gathered}
$$

voir [9], où $\mathcal{H}=\frac{1}{2} b_{\alpha}^{\alpha}$ est la courbure moyenne de l'interface $\Sigma$ et où $D_{\alpha}$ est la dérivée covariante sur $\Sigma$. La composante surfacique du terme d'ordre 3 est aussi calculée dans [9].

On calcule ensuite les profils $\mathbf{V}_{j}$ de (5) à partir de la première équation de (1) écrite en paramétrisation normale et des profils $\mathbf{W}_{j}$. L'opérateur rotationnel en paramétrisation normale admet la décomposition suivante en composantes contravariantes, voir [9]

$$
(\nabla \times \mathbf{E})^{\alpha}=\epsilon^{3 \beta \alpha}\left(\partial_{3}^{h} \mathrm{E}_{\beta}-\partial_{\beta} \mathrm{E}_{3}\right) \quad \text { et } \quad(\nabla \times \mathbf{E})^{3}=\epsilon^{3 \alpha \beta} D_{\alpha}^{h} \mathrm{E}_{\beta} \quad \text { sur } \quad \Sigma_{h}
$$

Ici $D_{\alpha}^{h}$ est la dérivée covariante sur $\Sigma_{h}$, surface moyenne de hauteur $h:=y_{3}$ par rapport à $\Sigma$ dans le conducteur; $\epsilon$ est le tenseur de Levi-Civita, voir [5,3], dont les composantes contravariantes $\epsilon^{i j k}$ dépendent de $h$ et s'expriment dans un système de coordonnées normales

$$
\epsilon^{i j k}={\sqrt{\operatorname{det}\left(a_{\alpha \beta}(h)\right)}}^{-1} \epsilon_{0}(i, j, k),
$$

où $a_{\alpha \beta}(h)$ désigne le tenseur métrique sur la variété $\Sigma_{h}$, les indices $i, j, k \in\{1,2,3\}$ et $\epsilon_{0}(i, j, k)=1$ si $(i, j, k)$ est une permutation circulaire directe, $\epsilon_{0}(i, j, k)=-1 \mathrm{si}(i, j, k)$ est une permutation circulaire indirecte et $\epsilon_{0}(i, j, k)=0$ sinon.

Remarque 1 D'après [8,2], on a

donc, si $a=\operatorname{det}\left(a_{\alpha \beta}\right)$, il vient (voir [9])

$$
a_{\alpha \beta}(h)=a_{\alpha \beta}-2 b_{\alpha \beta} h+b_{\alpha}^{\gamma} b_{\gamma \beta} h^{2},
$$

$$
\epsilon^{i j k}=\sqrt{a}^{-1}\left(1-2 \mathcal{H} h+O\left(h^{2}\right)\right) \epsilon_{0}(i, j, k) .
$$

Après le changement d'échelle $h=\delta Y_{3}$ puis développement selon les puissances de $\delta$ des équations (9), on déduit des développements (3) et (4) l'expression des premiers termes du développement (5) en champ magnétique (calculs contravariants) :

$$
\mathbf{V}_{0}\left(y_{\beta}, Y_{3}\right)=\mathbf{h}_{0}\left(y_{\beta}\right) \mathrm{e}^{-\lambda Y_{3}}
$$

où $\mathbf{h}_{0}\left(y_{\beta}\right)=\left(\mathbf{n} \times \mathbf{H}_{0}^{+}\right) \times \mathbf{n}\left(y_{\beta}, 0\right)$ est la composante tangentielle du terme $\mathbf{H}_{0}^{+}$, et si $\mathrm{h}_{j}^{\alpha}\left(y_{\beta}\right):=\left(\mathbf{H}_{j}^{+}\right)^{\alpha}\left(y_{\beta}, 0\right)$,

$$
\begin{gathered}
\mathcal{V}_{1}^{\alpha}\left(y_{\beta}, Y_{3}\right)=\left[\mathrm{h}_{1}^{\alpha}+Y_{3}\left(\mathcal{H} \mathrm{h}_{0}^{\alpha}+b_{\sigma}^{\alpha} \mathrm{h}_{0}^{\sigma}\right)\right]\left(y_{\beta}\right) \mathrm{e}^{-\lambda Y_{3}}, \\
v_{1}\left(y_{\beta}, Y_{3}\right)=\lambda^{-1} D_{\alpha} \mathrm{h}_{0}^{\alpha}\left(y_{\beta}\right) \mathrm{e}^{-\lambda Y_{3}} .
\end{gathered}
$$

On peut calculer les composantes covariantes $\mathcal{V}_{\alpha, 1}$ en utilisant le développement (10) de la métrique et les termes $\mathcal{V}_{0}^{\alpha}$ et $\mathcal{V}_{1}^{\alpha}$. Si $\mathrm{h}_{\alpha, j}\left(y_{\beta}\right):=\left(\mathbf{H}_{j}^{+}\right)_{\alpha}\left(y_{\beta}, 0\right)$, il vient

$$
\mathcal{V}_{\alpha, 1}\left(y_{\beta}, Y_{3}\right)=\left[\mathrm{h}_{\alpha, 1}+Y_{3}\left(\mathcal{H} \mathrm{h}_{\alpha, 0}-b_{\alpha}^{\sigma} \mathrm{h}_{\sigma, 0}\right)\right]\left(y_{\beta}\right) \mathrm{e}^{-\lambda Y_{3}}
$$




\section{Comparaison avec les résultats issus de [4]}

On compare dans ce paragraphe les premiers termes profils (7), (8), (11) et (12) avec ceux qu'on note $\widetilde{\mathcal{W}}_{\alpha, 1}$, $\widetilde{\mathcal{W}}_{\alpha, 2}, \widetilde{\mathbf{V}}_{0}$ et $\widetilde{\mathbf{V}}_{1}$, issus des formules (5.23), (5.27), (5.28) dans [4] pour une étude analogue des équations (1) et une condition au bord d'impédance. Les termes $\widetilde{\mathbf{V}}_{0}$ et $\widetilde{\mathcal{W}}_{\alpha, 1}$ sont cohérents avec (11) et (7) respectivement. Cependant des termes supplémentaires de courbure $b_{\sigma}^{\alpha}$ apparaissent dans les expressions (12) et (8) par rapport à leurs homologues $\widetilde{\mathcal{V}}_{1}^{\alpha}$ et $\widetilde{\mathcal{W}}_{\alpha, 2}$. De fait les termes $\widetilde{\mathcal{W}}_{\alpha}$ et $\mathcal{W}_{\alpha}$ sont les composantes d'un même champ de 1-forme sur $\Sigma_{h}$ vu comme champ de $\mathbb{R}^{3}$ mais calculés dans des bases différentes. On a $\mathcal{W}_{\alpha}(h) d y^{\alpha}(h)=\widetilde{\mathcal{W}}_{\alpha}(h) d y^{\alpha}(0)$ où $d y^{\alpha}(h)$ et $d y^{\alpha}(0)$ sont des bases locales des plans cotangent (parallèles) à $\Sigma_{h}$ et $\Sigma=\Sigma_{0} \subset \mathbb{R}^{3}$ respectivement. Le lien entre ces deux composantes est donné par le shifter, voir [8] et [2, §2.D], et on a

$$
\widetilde{\mathcal{W}}_{\alpha}=\mathcal{W}_{\alpha}-h b_{\alpha}^{\beta} \mathcal{W}_{\beta} \quad \text { et } \quad \mathcal{W}_{\alpha}=\sum_{n \geqslant 0} h^{n}\left(b^{n}\right)_{\alpha}^{\beta} \widetilde{\mathcal{W}}_{\beta}
$$

où $\left(b^{n}\right)_{\alpha}^{\beta}=b_{\nu_{1}}^{\beta} b_{\nu_{2}}^{\nu_{1}} \cdots b_{\alpha}^{\nu_{n-1}}$ avec la convention $\left(b^{0}\right)_{\alpha}^{\beta}=\delta_{\alpha}^{\beta}$.

\section{Développement asymptotique de l'épaisseur de peau}

Dans un modèle 1D de l'effet de peau où le conducteur est modélisé par le demi-espace $z>0$, l'amplitude du champ électrique en tant qu'onde plane s'écrit :

$$
\|\mathbf{E}(z)\|=E_{0} \mathrm{e}^{-z / \ell(\sigma)},
$$

où $E_{0} \in \mathbb{R}$ et la quantité $\ell(\sigma)$ référencée dans la littérature comme épaisseur de peau est

$$
\ell(\sigma)=\sqrt{2 / \omega \mu_{0} \sigma} .
$$

Remarque 2 Les quantités $\ell(\sigma)$ et $\delta$ sont liées par la relation $\ell(\sigma)=\delta / \operatorname{Re} \lambda$.

Afin de définir l'épaisseur de peau associée à notre modèle 3D pour une donnée $\mathbf{j}$, on définit, à partir du champ magnétique

$$
\mathbf{V}_{(\delta)}\left(y_{\alpha}, y_{3}\right):=\mathbf{H}_{(\delta)}^{-}(\mathbf{x}), \quad y_{\alpha} \in \Sigma, \quad 0 \leq y_{3}<h_{0}\left(h_{0}>0 \text { assez petit }\right) .
$$

Définition 4.1 Soit $\Sigma$ une surface régulière et $\mathbf{j}$ une donnée du problème (1) telles que pour tout $y_{\alpha}$ dans $\Sigma$, $\left\|\mathbf{V}_{(\delta)}\left(y_{\alpha}, 0\right)\right\| \neq 0$. L'épaisseur de peau est la quantité $\mathcal{L}\left(\sigma, y_{\alpha}\right)$ définie sur $\Sigma$ ayant la plus petite valeur réelle positive telle que

$$
\left\|\mathbf{V}_{(\delta)}\left(y_{\alpha}, \mathcal{L}\left(\sigma, y_{\alpha}\right)\right)\right\|=\left\|\mathbf{V}_{(\delta)}\left(y_{\alpha}, 0\right)\right\| \mathrm{e}^{-1} .
$$

Remarque 3 D'après le théorème des valeurs intermédiaires, $\mathcal{L}\left(\sigma, y_{\alpha}\right)$ est bien définie et est intrinsèque.

Le théorème suivant précise le comportement de l'épaisseur de peau $\mathcal{L}\left(\sigma, y_{\alpha}\right)$ à haute conductivité $\sigma$ en tout point $y_{\alpha}$ de l'interface $\Sigma$.

Théorème 4.2 Soit $\Sigma$ une surface régulière de courbure moyenne $\mathcal{H}$. On suppose que $\mathbf{h}_{0}\left(y_{\alpha}\right) \neq 0$. L'épaisseur de peau $\mathcal{L}\left(\sigma, y_{\alpha}\right)$ admet le développement asymptotique suivant

$$
\mathcal{L}\left(\sigma, y_{\alpha}\right)=\ell(\sigma)\left(1+\mathcal{H}\left(y_{\alpha}\right) \ell(\sigma)+\mathcal{O}\left(\sigma^{-1}\right)\right), \quad \sigma \rightarrow \infty .
$$

PREUVE. La démonstration est effectuée par des calculs contravariants sur le champ $\mathbf{V}(\delta)$ défini par $\mathbf{V}(\delta)\left(y_{\alpha}, Y_{3}\right):=$ $\mathbf{V}_{(\delta)}\left(y_{\alpha}, y_{3}\right)$. D'après (5), on a

$$
\mathbf{V}(\delta)=\left(\mathcal{V}_{0}^{\alpha}, 0\right)+\delta\left(\mathcal{V}_{1}^{\alpha}, v_{1}\right)+\mathcal{O}\left(\delta^{2}\right)
$$

Par définition du module d'un champ de vecteurs il vient :

$$
\|\mathbf{V}(\delta)\|^{2}=a_{\alpha \beta}(h) \mathcal{V}_{0}^{\alpha} \overline{\mathcal{V}_{0}^{\beta}}+2 \delta a_{\alpha \beta}(h) \operatorname{Re} \mathcal{V}_{0}^{\alpha} \overline{\mathcal{V}_{1}^{\beta}}+\mathcal{O}\left(\delta^{2}\right)
$$


D’où en développant la métrique, voir (10), puis en utilisant (11) et (12)

$$
\begin{aligned}
\|\mathbf{V}(\delta)\|^{2} & =\left(a_{\alpha \beta}-2 \delta Y_{3} b_{\alpha \beta}\right) \mathcal{V}_{0}^{\alpha} \overline{\mathcal{V}_{0}^{\beta}}+2 \delta a_{\alpha \beta} \operatorname{Re} \mathcal{V}_{0}^{\alpha} \overline{\mathcal{V}_{1}^{\beta}}+\mathcal{O}\left(\left(\delta+y_{3}\right)^{2}\right) \\
& =\left[\left\|\mathbf{h}_{0}\right\|^{2}-2 \delta Y_{3} b_{\alpha \beta} \mathrm{h}_{0}^{\alpha} \overline{\mathrm{h}_{0}^{\beta}}+2 \delta a_{\alpha \beta} \operatorname{Re}_{0}^{\alpha}\left(\overline{\mathrm{h}_{1}^{\beta}}+Y_{3}\left(\mathcal{H} \overline{\mathrm{h}_{0}^{\beta}}+b_{\sigma}^{\beta} \overline{\mathrm{h}_{0}^{\sigma}}\right)\right)+\mathcal{O}\left(\left(\delta+y_{3}\right)^{2}\right)\right] \mathrm{e}^{-2 \operatorname{Re}(\lambda) Y_{3}} \\
& =\left[\left\|\mathbf{h}_{0}\right\|^{2}+2 \delta \operatorname{Re}\left\langle\mathbf{h}_{0}, \mathbf{h}_{1}\right\rangle+2 \delta Y_{3} \mathcal{H}\left\|\mathbf{h}_{0}\right\|^{2}+\mathcal{O}\left(\left(\delta+y_{3}\right)^{2}\right)\right] \mathrm{e}^{-2 \operatorname{Re}(\lambda) Y_{3}} .
\end{aligned}
$$

Comme $\mathbf{h}_{0}\left(y_{\alpha}\right) \neq 0$, on peut définir $m\left(y_{\alpha}, y_{3} ; \delta\right)$ de façon que

$$
\left\|\mathbf{V}_{(\delta)}\left(y_{\alpha}, y_{3}\right)\right\|^{2}=\left\|\mathbf{h}_{0}\left(y_{\alpha}\right)\right\|^{2} m\left(y_{\alpha}, y_{3} ; \delta\right) \text {. }
$$

D’après l'identité ci-dessus, on a

$$
m\left(y_{\alpha}, y_{3} ; \delta\right)=\left[1+2 \delta\left\|\mathbf{h}_{0}\left(y_{\alpha}\right)\right\|^{-2} \operatorname{Re}\left\langle\mathbf{h}_{0}\left(y_{\alpha}\right), \mathbf{h}_{1}\left(y_{\alpha}\right)\right\rangle+2 y_{3} \mathcal{H}\left(y_{\alpha}\right)+\mathcal{O}\left(\left(\delta+y_{3}\right)^{2}\right)\right] \mathrm{e}^{-2 y_{3} \operatorname{Re}(\lambda) / \delta}
$$

et, ainsi

$$
m\left(y_{\alpha}, 0 ; \delta\right)=1+2 \delta\left\|\mathbf{h}_{0}\left(y_{\alpha}\right)\right\|^{-2} \operatorname{Re}\left\langle\mathbf{h}_{0}\left(y_{\alpha}\right), \mathbf{h}_{1}\left(y_{\alpha}\right)\right\rangle+\mathcal{O}\left(\delta^{2}\right) .
$$

Par définition de $\mathcal{L}\left(\sigma, y_{\alpha}\right)$, on a

$$
m\left(y_{\alpha}, \mathcal{L}\left(\sigma, y_{\alpha}\right) ; \delta\right) / m\left(y_{\alpha}, 0 ; \delta\right)=\mathrm{e}^{-2} .
$$

Par un développement asymptotique en $\delta$, on en déduit (15).

Remarque 4 (i) Si on pose

$$
\mathbf{W}_{(\delta)}\left(y_{\alpha}, y_{3}\right):=\mathbf{E}_{(\delta)}^{-}(\mathbf{x}), \quad y_{\alpha} \in \Sigma, \quad 0 \leq y_{3}<h_{0},
$$

on peut définir une épaisseur de peau similairement à (14) à partir du champ électrique $\mathbf{W}_{(\delta)}$. On peut montrer le même développement asymptotique (15) pour cette nouvelle épaisseur de peau.

(ii) Si on pousse plus loin le développement asymptotique de $\mathcal{L}\left(\sigma, y_{\alpha}\right)$ on trouve des termes qui dépendent du second membre du problème (1) et non plus seulement de la courbure moyenne du bord du conducteur.

\section{Références}

[1] G. Caloz, M. Dauge, V. Péron. Uniform estimates for transmission problems with high contrast in heat conduction and electromagnetism. Prépublication IRMAR 09-40, Rennes, 2009. http ://hal.archives-ouvertes.fr/hal-00422315/en/

[2] E. FAOU. Elasticity on a thin shell : formal series solution. Asymptot. Anal., 31(3-4) :317-361, 2002.

[3] H. Goldstein. Classical mechanics. Addison-Wesley Publishing Co., Reading, Mass., second edition, 1980. Addison-Wesley Series in Physics.

[4] H. Haddar, P. Joly, H.-M. NGUYen. Generalized impedance boundary conditions for scattering problems from strongly absorbing obstacles : the case of Maxwell's equations. Math. Models Methods Appl. Sci. 18(10) (2008) 1787-1827.

[5] T. Levi-Civita. The absolute differential calculus. Dover Phoenix Editions. Dover Publications Inc., Mineola, NY, 2005. Calculus of tensors, Translated from the Italian by Marjorie Long, Edited by Enrico Persico, Reprint of the 1926 translation.

[6] R.C. MacCamy, E. Stephan. Solution procedures for three-dimensional eddy current problems. J. Math. Anal. Appl. 101(2) (1984) 348-379.

[7] R.C. MacCamy, E. Stephan. A skin effect approximation for eddy current problems. Arch. Rational Mech. Anal. 90(1) (1985) 87-98.

[8] P. M. NAGHDi. Foundations of elastic shell theory. Progress in Solid Mechanics, 4, North-Holland, Amsterdam (1963) 1-90.

[9] V. PÉRON. Modélisation mathématique de phénomènes électromagnétiques dans des matériaux à fort contraste. Thèse. Université de Rennes 1, 2009. http ://tel.archives-ouvertes.fr/tel-00421736/fr/.

[10] E. STEPhan. Solution procedures for interface problems in acoustics and electromagnetics. In Theoretical acoustics and numerical techniques, volume 277 of CISM Courses and Lectures, pages 291-348. Springer, Vienna 1983. 